\title{
Performance Measure of Fuzzy Simple Queue with Balking and Feedback Customers using Symmetric Heptagonal Fuzzy Numbers
}

\author{
M. S. El -Paoumy \\ Department of Statistics, \\ Faculty of Commerce, Al-Azhar University, Dakahlia, Egypt
}

\begin{abstract}
This paper investigates the simple queue with balking and feedback customers by using the fuzzy set theory. The main proposal is a mathematical programming approach to develop the membership function of the performance measures of the model, in which the arrival rate and service rate are defined as fuzzy numbers. The Zadeh's extension principle and $\alpha$-cut approach are used to transform fuzzy queue into a family of crisps queue. To illustrate the approach, a numerical example is presented for heptagonal fuzzy numbers. Also, economic analysis of system is carried out by developing a cost model when some of parameters of the system are fuzzy numbers.
\end{abstract}

\section{General Terms}

Fuzzy set theory - Simple queue.

\section{Keywords}

Feedback queue; Simple queue; Symmetric heptagonal fuzzy number.

\section{INTRODUCTION}

The queueing theory treats some of the unavoidable situations in our daily life (Hospital, Bank, Reservation counter, etc.). The economic analysis of queueing model is a very interesting area of investigation.

Thus, fuzzy queueing models have been described by a many researchers such as S. U. Malini and Felbin [6], Mueen et al. [4], Rajalakshmi and Julia[2], Usha and Chanda[3] and Noor et al. [5]. Fuzzy problem has been studied by using heptagonal fuzzy number by Shanmugasundaram and Thamilselvi [8]. Zadeh [1] introduced the main concepts of fuzziness. R.J. Lie and E.S. Lee are the first scientists to study the fuzzy queueing model in 1989. This model was also developed by many researchers, such as J. J. Buckely [9] in 1990, R.S. Negi and E.S. Lee [10] in 1992, S.P. Chen [12] in 2005 and R. Srinivasan [11] in 2014. In this work, a single-server Markovian queueing system with balking and feedback customers is considered.

The aim of this work is to discuss single server queueing system with two concepts, namely, balking and feedback. The customer is served as classical discipline (FCFS) using heptagonal fuzzy numbers under -cut representation through DSW (Dong, Shah, Wong) algorithm to define a membership function of the performance measures in single server fuzzy queueing model. Also, economic analysis of system is presented by developing a cost model when some of parameters of the system are fuzzy numbers.

\section{FUZZY SIMPLE QUEUE WITH BALKING AND FEEDBACK CUSTOMERS}

Consider a single server fuzzy queueig model with balking and feedback customers, in which:

1- Customers arrive at the server one by one according to Poisson process with rate $\lambda_{n}$, where

$\lambda_{n}=\left\{\begin{array}{l}\lambda, n=0 \\ \beta \lambda, n \geq 1\end{array}\right.$,

where $\beta=\operatorname{Pr}\{$ that customer joins the queue $\}$.

2- The customers served by one server with rate $\mu$ according to classical discipline (FIFO).

3- After completion of each service the customer either joins at the end of the queue as feedback customer with probability $(1-\zeta)$ or departure the system with probability $\zeta$.

\subsection{Basic definitions of fuzzy sets theory}

In this part, some basic concepts of fuzzy sets are shown.

Definition 2.1: Let $T$ be a classical set or a universe, a fuzzy subset $\tilde{A}$ (or a fuzzy set $\tilde{A}$ ) in $T$ defined by the function $\phi_{\tilde{A}}(\mathrm{t})$, called membership function of $\tilde{A}$ from $T$ to the real unit interval $[0,1]$. Note that $\phi_{\tilde{A}}(\mathrm{t})$ is called the grade or the membership degree of $t, \forall t \in \tilde{A}$.

Definition 2.2: Let $\tilde{A}$ be a subset in the universe $T$, the $\alpha$ -cut of $\tilde{A}$ denoted by $\tilde{A}_{\alpha}$ is a classical set defined as follows: $\tilde{A}_{\alpha}=\left\{\mathrm{t} \in T / \phi_{\tilde{A}}(\mathrm{t}) \geq \alpha\right\}$

Definition 2.3: Let $\tilde{A}$ be a subset in universe $T$ the support $(\operatorname{supp}(\tilde{A}))$ and the height $(\operatorname{hgt}(\tilde{A}))$ of $\tilde{A}$ are the crisp sets defined respectively as follows:

$$
\begin{aligned}
& \operatorname{supp}(\tilde{A})=\left\{t \in T / \phi_{\tilde{A}}(t)>0\right\} \\
& \operatorname{hgt}(\tilde{A})=\max \left\{\phi_{\tilde{A}}(t) / t \in T\right\}
\end{aligned}
$$


Definition 2.4: A fuzzy set $\tilde{A}$ is said to normal if and only if $\operatorname{hgt}(\tilde{A})=1$, and convex if and only if $\phi_{\tilde{A}}(\delta s+(1-\delta) t) \geq \min \left\{\phi_{\tilde{A}}(s), \phi_{\tilde{A}}(t)\right\}$.

\subsection{Fuzzy number}

Definition 2.5: A fuzzy number set $\tilde{A}$ in the inverse $T$ is a fuzzy number if and only if it satisfies the following conditions:
1) $T=\mathfrak{R}$
2) $\tilde{A}_{\text {is normal. }}$
3) $\tilde{A}$ is convex.
4) the membership function $\phi_{\tilde{A}}(\mathrm{t})$ is piecewise continuous.
5) there exist one and only one $t \in \mathfrak{R}$ such that $\phi_{\tilde{A}}(\mathrm{t})=1$.

From this definition of the fuzzy number can be represented in more forms as Triangular, Trapezoidal, Pentagonal, Hexagonal, Heptagonal, and Octagonal etc.

\subsection{Heptagonal fuzzy number (H F N)}

Definition 2.6: A symmetric fuzzy number $\tilde{A}$ is a normal HFN defined by $\tilde{A}=\left(a_{1}, a_{2}, \ldots, a_{7}\right)$ where $a_{i} \in \mathfrak{R}$, $i=1,2, \ldots, 7$ and its membership function[7].

$\phi_{\tilde{A}}(\mathrm{t})= \begin{cases}0, & \text { if } t<a_{1} \\ r\left(\frac{t-a_{1}}{a_{2}-a_{1}}\right), & \text { if } a_{1} \leq t \leq a_{2} \\ r, & \text { if } a_{2} \leq t \leq a_{3} \\ r+(1-r)\left(\frac{t-a_{3}}{a_{4}-a_{3}}\right), & \text { if } a_{3} \leq t \leq a_{4} \\ r+(1-r)\left(\frac{a_{5}-t}{a_{5}-a_{4}}\right), & \text { if } a_{4} \leq t \leq a_{5} \\ r, & \text { if } a_{5} \leq t \leq a_{6} \\ r\left(\frac{a_{7}-t}{a_{7}-a_{6}}\right), & \text { if } a_{6} \leq t \leq a_{7} \\ 0, & \text { if } t \geq a_{7} .\end{cases}$

where $0<r<1$.

Figure 1 shows that the symmetric $\mathrm{HpFN}$ has a Heptagonal shape with seven vertices $\tilde{A}=\left(a_{1}, a_{2}, \ldots, a_{7}, r, 1\right)$.

Definition2.7: If $\tilde{A}$ is a HpFNs, then $\alpha$-cut of $\tilde{A}$ is

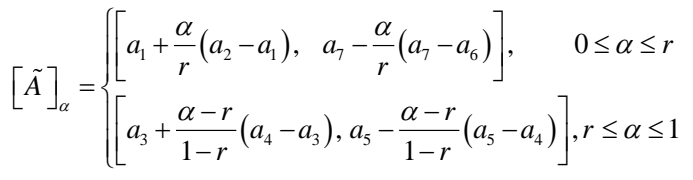

\section{PERFORMANCE MEASURES OF FUZZY QUEUE AND COST MODEL}

In this section, some performance measures of the system are given. By using these performance measures, the economics of the system are investigated.

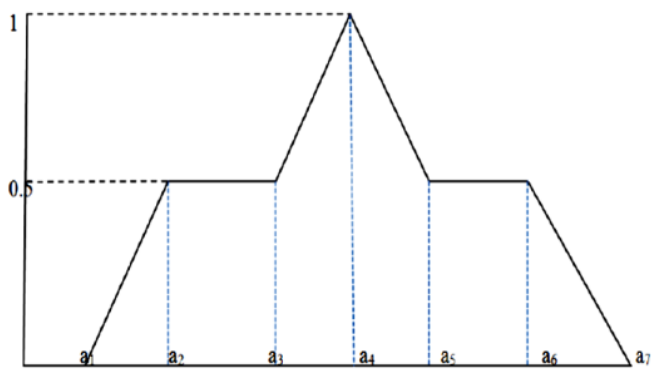

Fig 1: Graphical description of the HpFNs.

\subsection{Performance measures}

Consider a simple fuzzy queuing system with single server, denoted by $F M / F M / 1$ in which arrivals have a Poisson distribution with fuzzy rate $\tilde{\lambda}$ and service time according to exponential distribution with fuzzy rate $\tilde{\mu}$. The service discipline is (FIFO) and the waiting room with infinite capacity.

The arrival and service rates are defined as

$$
\begin{aligned}
& \tilde{\lambda}=\left\{\left(t, \phi_{\tilde{\lambda}}(t) / t \in T\right\},\right. \\
& \tilde{\mu}=\left\{\left(\mathrm{s}, \phi_{\tilde{\mu}}(\mathrm{s}) / \mathrm{s} \in S\right\},\right.
\end{aligned}
$$

where $T, S$ are crisp rate and $\phi_{\tilde{\lambda}}(t), \phi_{\tilde{\mu}}(s)$ are the corresponding membership function for the following performance measures of $F M / F M / 1$ system :

The mean number of customer in the system is

$$
\tilde{L}=\frac{\beta \tilde{\lambda}}{\zeta \tilde{\mu}-\beta \tilde{\lambda}}
$$

The mean waiting time in the system is

$$
\tilde{W}=\frac{\tilde{L}}{\beta \tilde{\lambda}}=\frac{1}{\zeta \tilde{\mu}-\beta \tilde{\lambda}} .
$$

The empty probability of system is

$$
\tilde{P}_{0}=\frac{\zeta \tilde{\mu}-\beta \tilde{\lambda}}{\zeta \tilde{\mu}}
$$

The average rate of balking is 


$$
B . \tilde{R}=\tilde{\lambda}(1-\beta)\left(1-\tilde{P}_{0}\right)=\frac{\beta(1-\beta) \tilde{\lambda}^{2}}{\zeta \tilde{\mu}}
$$

\subsection{Cost Model}

In this section, cost analysis of $F M / F M / 1$ queue according the arrival rate and service rate are fuzzy numbers are studied.

In order to approximating the function of fuzzy Total Expected cost $(T E \tilde{C})$, Total fuzzy Expected Revenue ( $T E \tilde{R}$ ), and Total fuzzy Expected Profit $(T E \tilde{P})$, the following formulas are used in this cost model:

$c_{h}$ : Holding cost per unit per unit time.

$c_{s}:$ Cost per service per unit time.

$c_{l}$ : Cost related with each lost unit per unit time.

$R:$ Revenue earned per unit time.

Cost model function is as follows:

$T E \tilde{C}=c_{h} \tilde{L}+c_{l} \beta \tilde{\lambda}\left(1-\tilde{P}_{0}\right)+c_{s} \zeta \tilde{\mu}$,

$T E \tilde{R}=R \zeta \tilde{\mu}\left(1-\tilde{P}_{0}\right)$,

$T E \tilde{P}=T E \tilde{R}-T E \tilde{C}$

\section{ARITHMETIC OPERATIONS ON INTERVALS}

For any two intervals $\left[b_{1}, b_{2}\right]$ and $\left[b_{3}, b_{4}\right]$, the operations on two intervals are given below.

1. $\left[b_{1}, b_{2}\right]+\left[b_{3}, b_{4}\right]=\left[b_{1}+\mathrm{b}_{3}, b_{2}+b_{4}\right]$

2. $\left[b_{1}, b_{2}\right]-\left[b_{3}, b_{4}\right]=\left[b_{1}-\mathrm{b}_{4}, b_{2}-b_{3}\right]$

3. $\left[b_{1}, b_{2}\right] *\left[b_{3}, b_{4}\right]=$

$\left[\min \left(b_{1} b_{3}, b_{1} b_{4}, b_{2} \mathrm{~b}_{3}, b_{2} b_{4}\right), \max \left(b_{1} b_{3}, b_{1} b_{4}, b_{2} \mathrm{~b}_{3}, b_{2} b_{4}\right)\right]$

4. $\left[b_{1}, b_{2}\right] \div\left[b_{3}, b_{4}\right]=\left[\frac{b_{1}}{b_{4}}, \frac{b_{2}}{b_{3}}\right]$ provided that

$$
0 \notin\left[b_{3}, b_{4}\right]
$$

5. $a\left[b_{1}, b_{2}\right]=\left[a b_{1}, a b_{2}\right], a>0$

$$
=\left[a b_{2}, a b_{1}\right], a<0
$$

as in Mohammed Shapique [7].

\section{SOLUTION PROCEDURE FOR PROBLEM}

The algorithm for approximate method, given $\alpha$ - cut levels, consists of the following steps:

1. Taking $\alpha$ value from $0,0.1,0.2, \ldots, 1$.
2. For selected $\alpha$ value from step 1, find the intervals in the input membership function corresponding to it.

3. Compute the interval for the output membership function for $\alpha$-cut by using standard binary operations on interval.

4. Repeat the steps from 1 to 3 for various values of $\alpha$-cut to get the solution.

\section{NUMERICAL EXAMPLE}

For the queue FM / FM /1 with balking and feedback, where the arrival rate and service rate are $\mathrm{HpFNs}$ represented by

$$
\tilde{\lambda}=[3,4,5,6,7,8,9]
$$

and

$\tilde{\mu}=[13,14,15,16,17,18,19]$ per hour, respectively, also $r=0.5, c_{h}=5, c_{s}=6, c_{l}=12, R=100$, $\beta=0.2$ and $\zeta=0.3$.

In $F M / F M / 1$ queue, by taking the fuzzy number for arrival rate and service rate, then

$$
\begin{aligned}
& {[\tilde{L}]_{\alpha}=\frac{X}{Y-X},} \\
& {[\tilde{W}]_{\alpha}=\frac{[\tilde{L}]_{\alpha}}{X}=\frac{1}{Y-X},} \\
& \text { and }[\tilde{P}]_{\alpha}=\frac{Y-X}{Y},
\end{aligned}
$$

where $X$ and $Y$ are $\alpha$-cut for $\beta \tilde{\lambda}$ and $\zeta \tilde{\mu}$ are given by

$$
X=\beta[\tilde{\lambda}]_{\alpha}=\left\{\begin{array}{l}
{\left[0.2\left(3+\left(\frac{\alpha}{0.5}\right)\right), 0.2\left(9-\left(\frac{\alpha}{0.5}\right)\right)\right], 0 \leq \alpha \leq 0.5} \\
{\left[0.2\left(5+\left(\frac{\alpha-0.5}{0.5}\right)\right), 0.2\left(7-\left(\frac{\alpha-0.5}{0.5}\right)\right)\right], 0.5 \leq \alpha \leq 1}
\end{array}\right.
$$

$$
Y=\zeta[\tilde{\mu}]_{\alpha}= \begin{cases}{\left[0.3\left(13+\left(\frac{\alpha}{0.5}\right)\right), 0.3\left(19-\left(\frac{\alpha}{0.5}\right)\right)\right],} & 0 \leq \alpha \leq 0.5 \\ {\left[0.3\left(15+\left(\frac{\alpha-0.5}{0.5}\right)\right), 0.3\left(17-\left(\frac{\alpha-0.5}{0.5}\right)\right)\right], 0.5 \leq \alpha \leq 1}\end{cases}
$$

To compute the $\alpha$-cut for $\tilde{W}, \tilde{L}$, and $T E \tilde{P}$, the following calculations are carried out

$$
Y-X=\left\{\begin{array}{l}
{[2.1+\alpha, 5.1-\alpha], 0 \leq \alpha \leq 0.5} \\
{[3.1+(\alpha-0.5), 4.1-(\alpha-0.5)], 0.5 \leq \alpha \leq 1}
\end{array},\right.
$$

$$
1-\left[\tilde{P}_{0}\right]_{\alpha}=\frac{X}{Y}= \begin{cases}{\left[\frac{0.6+0.4 \alpha}{5.7-0.6 \alpha}, \frac{1.8-0.4 \alpha}{3.9+0.6 \alpha}\right],} & 0 \leq \alpha \leq 0.5 \\ {\left[\frac{1+0.4(\alpha-0.5)}{5.1-0.6(\alpha-0.5)}, \frac{1.4-0.4(\alpha-0.5)}{4.5+0.6(\alpha-0.5)}\right],} & 0.5 \leq \alpha \leq 1 .\end{cases}
$$


$[\tilde{L}]_{\alpha}=\frac{X}{Y-X}=\left\{\begin{array}{l}{\left[\frac{0.6+0.4 \alpha}{5.1-\alpha}, \frac{1.8-0.4 \alpha}{2.1+\alpha}\right], 0 \leq \alpha \leq 0.5} \\ {\left[\frac{0.8+0.4 \alpha}{4.6-\alpha}, \frac{1.6-0.4 \alpha}{2.6+\alpha}\right], 0.5 \leq \alpha \leq 1}\end{array}\right.$,

and

$[\tilde{W}]_{\alpha}=\frac{1}{Y-X}=\left\{\begin{array}{l}{\left[\frac{1}{5.1-\alpha}, \frac{1}{2.1+\alpha}\right], 0 \leq \alpha \leq 0.5} \\ {\left[\frac{1}{4.6-\alpha}, \frac{1}{2.6+\alpha}\right], 0.5 \leq \alpha \leq 1}\end{array}\right.$.

Hence

$$
[T E \tilde{P}]_{\alpha}=R * X-c_{h} * \frac{X}{Y-X}-c_{l} * X * \frac{X}{Y}-c_{s} * Y,
$$

and

$$
[T E \tilde{P}]_{\alpha}=\left\{\begin{array}{l}
{\left[\text { tep }_{\alpha}^{L 1}, t e p_{\alpha}^{U 1}\right], 0 \leq \alpha \leq 0.5} \\
{\left[t e p_{\alpha}^{L 2}, t e p_{\alpha}^{U 2}\right], 0.5 \leq \alpha \leq 1}
\end{array}\right.
$$

where

$t e p_{\alpha}^{L 1}=150(0.6+0.4 \alpha)-5\left(\frac{1.8-0.4 \alpha}{2.1+\alpha}\right)-6(5.7-0.6 \alpha)-12 g_{1}$,

$0 \leq \alpha \leq 0.5$

$t e p_{\alpha}^{U 1}=150(1.8-0.4 \alpha)-5\left(\frac{0.6+0.4 \alpha}{5.1-\alpha}\right)-6(3.9+0.6 \alpha)-12 g_{2}$,

$0 \leq \alpha \leq 0.5$

$t e p_{\alpha}^{L 2}=150(0.8+0.4 \alpha)-5\left(\frac{1.6-0.4 \alpha}{2.6+\alpha}\right)-6(5.4-0.6 \alpha)-12 f_{1}$,

$0.5 \leq \alpha \leq 1$

$t e p_{\alpha}^{U 2}=150(1.6-0.4 \alpha)-5\left(\frac{0.8+0.4 \alpha}{4.6-\alpha}\right)-6(4.2+0.6 \alpha)-12 f_{2}$,

$0.5 \leq \alpha \leq 1$

$g_{1}=\max E, g_{2}=\min E, f_{1}=\max F$,

$f_{2}=\min F$,

$E=\left\{\frac{(0.6+0.4 \alpha)^{2}}{5.7-0.6 \alpha}, \frac{(0.6+0.4 \alpha)(1.8-0.4 \alpha)}{3.9+0.6 \alpha}, \frac{(0.6+0.4 \alpha)(1.8-0.4 \alpha)}{5.7-0.6 \alpha}, \frac{(1.8-0.4 \alpha)^{2}}{3.9+0.6 \alpha}\right\}$, and

$F=\left\{\frac{(0.8+0.4 \alpha)^{2}}{5.4-0.6 \alpha}, \frac{(0.8+0.4 \alpha)(1.6-0.4 \alpha)}{4.2+0.6 \alpha}, \frac{(1.6-0.4 \alpha)(0.8+0.4 \alpha)}{5.4-0.6 \alpha}, \frac{(1.6-0.4 \alpha)^{2}}{4.2+0.6 \alpha}\right\}$.

From equation (22) at $\alpha=1$, then $\tilde{L}$ is $\frac{1}{3}$, while at

$\alpha=0$, then $\tilde{L}$ is inside the interval $\left[\frac{6}{51}, \frac{18}{21}\right]$. Also, from equation (23) at $\alpha=1, \tilde{W}$ equals $\frac{10}{36}$, while at $\alpha=0$, then $\tilde{W}$ is impossible to fall lower $\frac{10}{51}$ or upper $\frac{10}{21}$. This means that the fuzzy expected number in the system is approximately between $\frac{6}{51}$ and $\frac{18}{21}$, also the fuzzy expected waiting time in the system is approximately between $\frac{10}{51}$ and $\frac{10}{21}$. Similarly from equation (25) at $\alpha=1$, then $T E \tilde{P}$ is $\frac{1289}{15}$, while at $\alpha=0$, then

$T E \tilde{P}$ is impossible to fall lower $\frac{5253}{455}$ or upper $\frac{50147}{323}$

Figures (2-4) show results of numerical simulations.

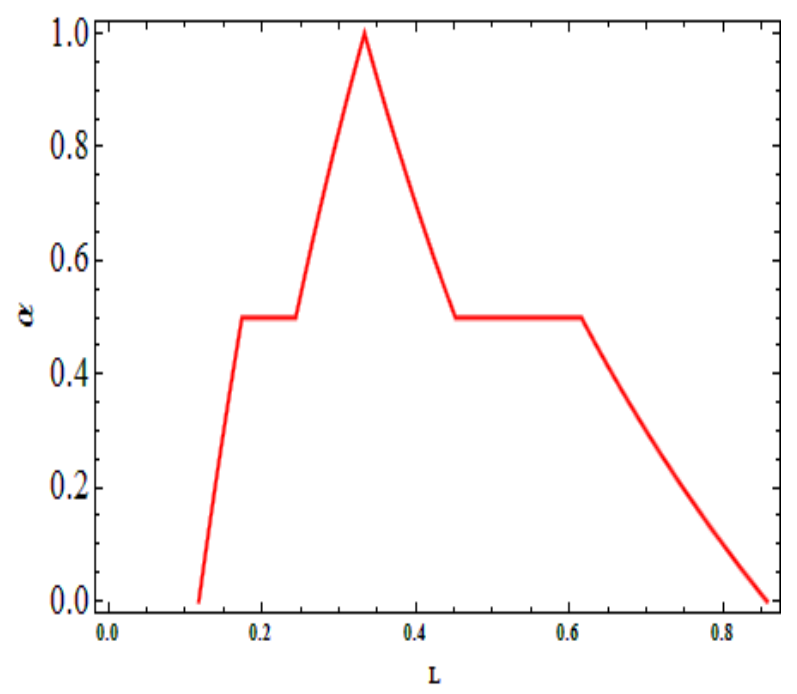

Fig 2: Membership function of the fuzzy mean number of customer in the system

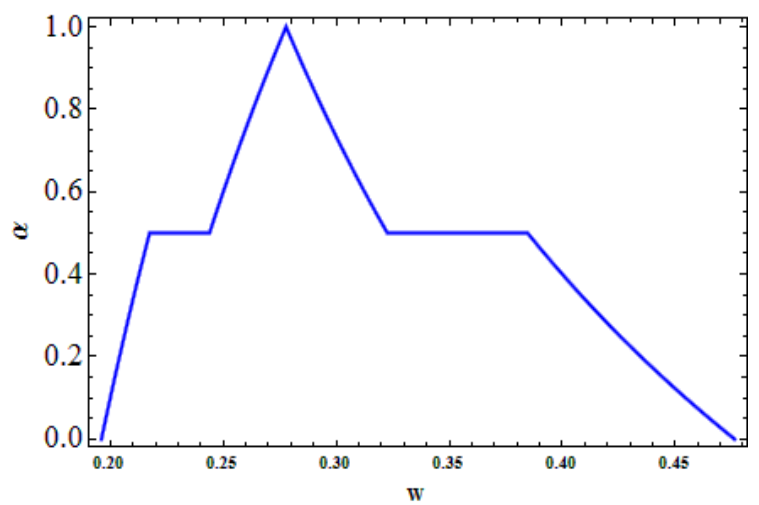

Fig 3: Membership function of the fuzzy mean waiting time in the system 


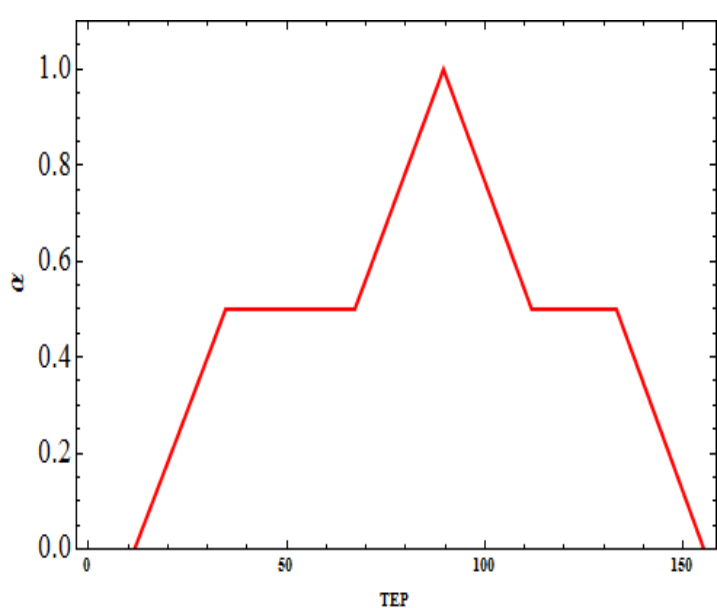

Fig 4: Membership function of the Total Fuzzy Expected Profit of the system

\section{SPECIAL CASES}

In the above described model taking $\beta=1$ and $\zeta=1$., we shall reach to an $F M / F M / 1$ fuzzy queuing system without balking and feedback of customers as studied by many researchers.

Put $\beta=1$ and $\zeta=1$ in equations (23) and (25), hence

$[\tilde{L}]_{\alpha}==\left\{\begin{array}{l}{\left[\frac{3+2 \alpha}{16-4 \alpha}, \frac{9-2 \alpha}{4+4 \alpha}\right], 0 \leq \alpha \leq 0.5} \\ {\left[\frac{4+2 \alpha}{14-4 \alpha}, \frac{8-2 \alpha}{6+4 \alpha}\right], 0.5 \leq \alpha \leq 1}\end{array}\right.$

and

$[\tilde{W}]_{\alpha}==\left\{\begin{array}{l}{\left[\frac{1}{16-4 \alpha}, \frac{1}{4+4 \alpha}\right], 0 \leq \alpha \leq 0.5} \\ {\left[\frac{1}{14-4 \alpha}, \frac{1}{6+4 \alpha}\right], 0.5 \leq \alpha \leq 1}\end{array}\right.$

Figure 5 and Figure 6 illustrate results of numerical simulations.

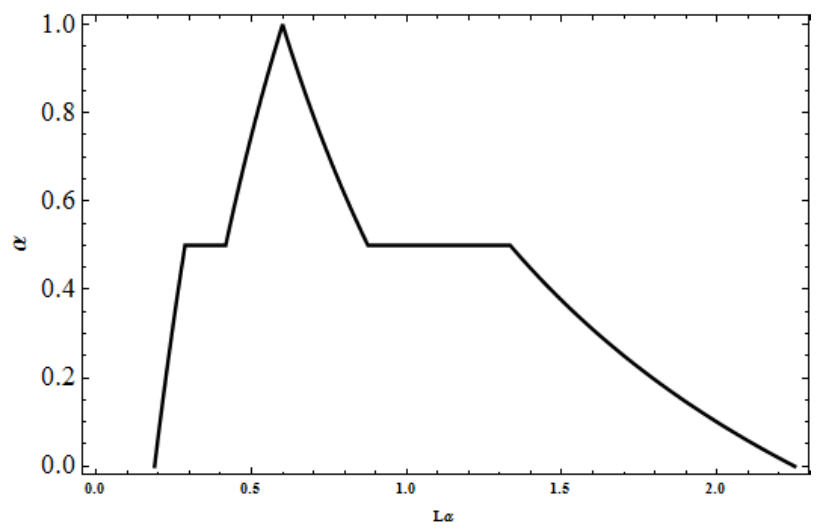

Fig 5: Membership function of the fuzzy mean number of customer in the system without balk and feedback customers

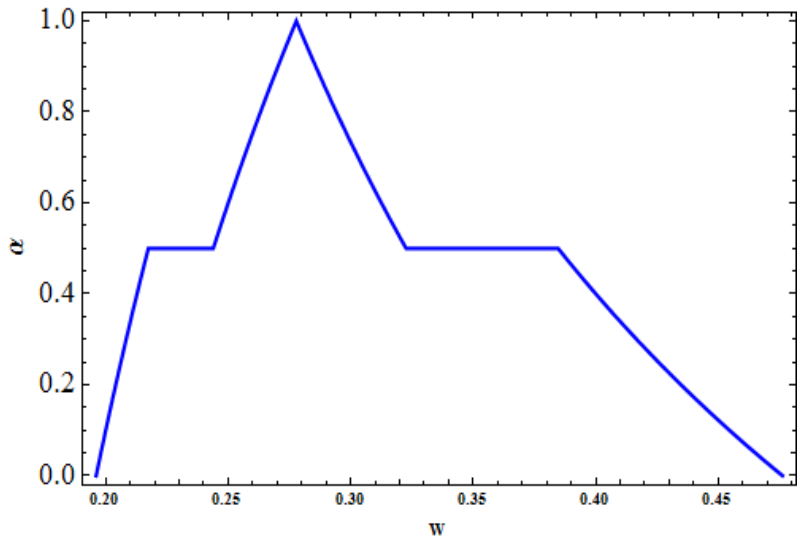

Fig 6: Membership function of the fuzzy mean waiting time in the system without balk and feedback customers

\section{CONCLUSION}

In this work, the fuzzy set of a single server queueing model with balking and feedback customers is discussed. The arrival rate and service rate are symmetric Heptagonal fuzzy numbers. The membership functions of waiting time in the system and mean number of customers are skewed left (nonsymmetric). From figure 2 and figure 3 , the membership functions of the fuzzy expected number in the system and the fuzzy expected waiting time in the system are skewed left, while figure 4 illustrates that the membership function of the total fuzzy expected Profit of the system is skewed right. The two systems with concepts balk and feedback customers are studied. The approximate values for the fuzzy expected mean of number in the system and the fuzzy expected waiting time in the system with concepts are found less than those of the system without concepts. Furthermore, HpFNs can be applied in various engineering and mathematical sciences.

\section{ACKNOWLEDGMENTS}

The author would like to thank the Editor and Reviewers for their helpful comments and suggestions.

\section{REFERENCES}

[1] L. F. Zadeh, " Fuzzy sets As a basic for a theory of possibility", Fuzzy sets system, vol. 1, pp. 3-28, 1978

[2] R, Rajalakshmi, K. Julia Mary, "Fuzzy analysis for finite capacity queueing model", International Journal of Applied Research, vol.3, No. 9, pp.103-106, 2017

[3] K. Usha Madhuri, K. Chandan, "Study on queueing system with Pentagon Fuzzy number using - cuts", International Journal of Advance Research, Ideas and Innovations in Technology, pp. 772-775, 2017

[4] Z. Mveen, R. Ramli. N. Z. Zaibidi, " Analysis of performance measures with single channel Fuzzy queues under two class by Ranking Method", Journal of Teleconmmunication, Electronic and Computer Engineering, vol. 9, No. 1,2 ,pp. 109-112, 2017

[5] M. Z. Noorhidayah, S. Aqilab, A. Nur, H. Nursu, A. Norani, "Comparison of queueing Performance using queueing theory model and fuzzy queueing model at check-in counter in airport", Mathematics and Statistics, vol. 7, No. 4A, pp. 17-23, 2019.

[6] S. U. Malini, Felbin, " An approach for solving fuzzy Transportation problem using Octagonal numbers ", Applied Mathematical Science, vol.7, No. 54, pp. 26612673, 2013 
[7] A. Mohammed Shapique, "Arithmetic Operations on Heptagonal Fuzzy Numbers", Asian Research Journal of Mathematics, 2(5): 1-25, 2017.

[8] S. Shanmugasundaram, K., Thamilselvi, " A study on single server queueing model using DSW Algorithm with Heptagonal and Octagonal fuzzy number", Global Journal of Pure and Applied Mathematics, vol. 14, No. 7, pp. 927 -936, 2018.

[9] J.J.Buckely " Elementery queueing theory based on possbility theory", Fuzzy set and systems, 37,43-52,
1990.

[10] D.S. Negi and E.S.Lee, " Analysis and simulation of fuzzy queue ", Fuzzy sets and systems, 46,321-330,1992.

[11] R. Srinivasan, "Fuzzy queueing model using DSW algorithm", International Journal of Advanced research in Mathematics and Application, vol. Issue 1, pp. 5762,204 .

[12] S.P. Chen, "Parametric nonlinear programming Approch to Fuzzy queues with bulk service", European Journal of Operation Research, 163, 434-444, 2005. 